\title{
ON BOUNDARY-DRIVEN TIME-DEPENDENT OSEEN FLOWS
}

\author{
PAUL DEURING \\ Laboratoire de Mathématiques Pures et Appliquées \\ Université du Littoral, B.P. 699, 62228 Calais, France \\ E-mail: Paul.Deuring@lmpa.univ-littoral.fr
}

\begin{abstract}
We consider the single layer potential associated to the fundamental solution of the time-dependent Oseen system. It is shown this potential belongs to $L^{2}\left(0, \infty, H^{1}(\Omega)^{3}\right)$ and to $H^{1}\left(0, \infty, V^{\prime}\right)$ if the layer function is in $L^{2}\left(\partial \Omega \times(0, \infty)^{3}\right)$. ( $\Omega$ denotes the complement of a bounded Lipschitz set; $V$ denotes the set of smooth solenoidal functions in $H_{0}^{1}(\Omega)^{3}$.) This result means that the usual weak solution of the time-dependent Oseen function with zero initial data and zero body force may be represented by a single layer potential, provided a certain integral equation involving the boundary data may be solved.
\end{abstract}

1. Introduction. Let $\Omega$ be an open set in $\mathbb{R}^{3}$ with compact complement and with Lipschitz boundary. (For the purposes of this article, we need not require that $\Omega$ is connected. Thus $\Omega$ is only supposed to be an exterior set, but not an exterior domain.) Put $Z_{T}:=\Omega \times(0, T)$ and $S_{T}:=\partial \Omega \times(0, T)$ for $T \in(0, \infty]$. Then consider the following initial-boundary value problem for the instationary Oseen system in $Z_{T}$ :

$$
\begin{gathered}
\partial_{t} u-\Delta_{x} u+\tau \cdot \partial_{x_{1}} u+\nabla_{x} \pi=f, \quad \operatorname{div}_{x} u=0 \text { in } Z_{T}, \\
u \mid S_{T}=b, \quad u(x, t) \rightarrow 0(|x| \rightarrow \infty) \text { for } t \in(0, T), \\
u(x, 0)=a(x) \quad \text { for } x \in \Omega,
\end{gathered}
$$

where the quantities $\tau \in(0, \infty)$ (Reynolds number) and $T \in(0, \infty]$ are given, as are the functions $f: Z_{T} \mapsto \mathbb{R}^{3}, a: \Omega \mapsto \mathbb{R}^{3}$ and $b: S_{T} \mapsto \mathbb{R}^{3}$. The velocity $u: Z_{T} \mapsto \mathbb{R}^{3}$ and the pressure $\pi: Z_{T} \mapsto \mathbb{R}$ are unknown.

In previous articles, problem (1) - (3) was usually solved by semigroup theory based on estimates of the Oseen resolvent ([4], [5], [8], [10]). Recently reference [2] proposed a potential theoretic approach which leads to solutions of (1) - (3) in the form of a sum of certain volume potentials plus a single-layer potential. This approach is useful

2000 Mathematics Subject Classification: Primary 76D07; Secondary 35Q30, 35K50, 35C15.

Key words and phrases: Oseen system, exterior domain, single-layer potential.

The paper is in final form and no version of it will be published elsewhere. 
for deriving pointwise decay estimates of exterior Oseen flows (see [2, Lemma 18]), and it may have other applications as well. For example, it may help to provide regularity results for Oseen flows in Lipschitz domains, in analogy to the theory developed by Shen [11] for evolutionary Stokes flows.

In the work at hand, we consider the single-layer potential appearing in the approach from [2]. This potential solves (1) - (3) with $f=0, a=0$, and may thus be considered as a boundary-driven Oseen flow. The velocity part of this potential, which we denote by $\mathcal{V}_{T}^{(\tau)}(\Phi)$ (see Section 2 for a precise definition), is a $C^{\infty}$-function in $\left(\mathbb{R}^{3} \backslash \partial \Omega\right) \times([0, T] \cap \mathbb{R})=$ $\left(\mathbb{R}^{3} \times([0, T] \cap \mathbb{R})\right) \backslash S_{T}$, for any layer function $\Phi \in L^{2}\left(0, T, L^{1}(\partial \Omega)^{3}\right)$ and for any $T \in(0, \infty]$. This follows immediately from the definition of $\mathcal{V}_{T}^{(\tau)}(\Phi)$ as a surface integral on $S_{T}$. In the present article we are interested in a property of $\mathcal{V}_{T}^{(\tau)}(\Phi)$ which is less obvious, namely $L^{2}$-integrability near $S_{T}$ and for large values of $|x|$ and $t$. In this respect, we will show that

$$
\begin{gathered}
\mathcal{V}_{T}^{(\tau)}(\Phi) \mid Z_{T} \in L^{\infty}\left(0, T, L^{2}(\Omega)^{3}\right), \quad \nabla\left(\mathcal{V}_{T}^{(\tau)}(\Phi) \mid Z_{T}\right) \in L^{2}\left(Z_{T}\right)^{9} \\
\partial_{t}\left(\mathcal{V}_{T}^{(\tau)}(\Phi) \mid Z_{T}\right) \in L^{2}\left(0, T, V^{\prime}\right) \quad \text { for } T \in(0, \infty], \Phi \in L^{2}\left(S_{T}\right)^{3}
\end{gathered}
$$

where $V$ is the space of solenoidal functions in $H_{0}^{1}(\Omega)^{3}$. Estimates of $\mathcal{V}_{T}^{(\tau)}(\Phi)$ corresponding to the relations in (4) will also be established; the right-hand sides of these estimates consist of the product a constant times $\|\Phi\|_{2}$, where the constant in question only depends on $\Omega$ and $\tau$, but not on $T$. Actually, we will admit functions $\Phi$ with somewhat less regularity than $L^{2}$-integrability on $S_{T}$; see Theorem 2.3 below for more details.

In order to indicate why this result is interesting, we recall that a weak solution of (1) - (3) is typically defined in such a way that the velocity belongs to the space $L^{2}\left(0, T, H^{1}(\Omega)^{3}\right) \cap H^{1}\left(0, T, V^{\prime}\right)$. We further recall this space is a uniqueness class for weak solutions to $(1)-(3)$ ([13, p. 172]). Thus our result means that a weak solution of (1) - (3) with $a=0$ and $f=0$ may be represented on $\Omega \times(0, T)$ by the single layer potential $\mathfrak{V}_{T}^{(\tau)}(\Phi)$, provided the layer function $\Phi \in L^{2}\left(S_{T}\right)^{3}$ solves the integral equation

$$
\mathfrak{V}_{T}^{(\tau)}(\Phi) \mid S_{T}=b
$$

Such a representation is useful in many respects. For example, it immediately implies that in the case $a=0, f=0$, a weak solution to (1) - (3) belongs to $C^{\infty}(\Omega \times(0, \infty))^{3}$. Or it yields decay results for $|x| \rightarrow \infty$; see [2, Lemma 18] for a simple example in this respect. But of course, all this is subordinate to solving the integral equation (5) on $S_{T}$ with unknown function $\Phi$. Some ideas on this problem may be found in [2, Section 3]. A more complete study of equation (5) is in preparation.

2. Notations and some auxiliary results. Main theorem. If $A \subset \mathbb{R}^{3}$, we write $A^{c}$ for the complement of $A$. The length $\alpha_{1}+\alpha_{2}+\alpha_{3}$ of a multi-index $\alpha \in \mathbb{N}_{0}^{3}$ is denoted by $|\alpha|_{1}$. We write $e_{1}$ for the unit vector $(1,0,0)$. For $r \in(0, \infty), x \in \mathbb{R}^{3}$, we write $B_{r}(x)$ for the open ball with centre $x$ and radius $r$. Put $B_{r}:=B_{r}(0)$.

The open set $\Omega \subset \mathbb{R}^{3}$ with compact complement $\Omega^{c}$ and with Lipschitz boundary $\partial \Omega$ will be kept fixed throughout. Without loss of generality, we may assume that $0 \in \Omega^{c}$ so that $|y| \leq \operatorname{diam} \Omega^{c}$ for $y \in \Omega^{c}$. We put $R_{0}:=4 \cdot \operatorname{diam} \Omega^{c}$, hence $\Omega^{c} \subset B_{R_{0} / 2}$. Recall the notations $Z_{T}:=\Omega \times(0, T)$ and $S_{T}:=\partial \Omega \times(0, T)$ for $T \in(0, \infty)$. For $R \in(0, \infty)$, 
we set $\Omega_{R}:=B_{R} \cap \Omega$. Let $H^{1}(\Omega)$ denote the usual Sobolev space of functions with weak first order derivatives in $L^{2}(\Omega)$. The usual norm of $H^{1}(\Omega)$ is denoted by \|\|$_{1,2}$, that is, $\|v\|_{1,2}:=\left(\|v\|_{2}^{2}+\sum_{k=1}^{3}\left\|\partial_{x_{k}} v\right\|_{2}^{2}\right)^{1 / 2}$ for $v \in H^{1}(\Omega)$. By $V$, we designate the closure of the set $\left\{\varphi \in C_{0}^{\infty}(\Omega)^{3}: \operatorname{div} \varphi=0\right\}$ in $H^{1}(\Omega)^{3}$. We define $V^{\prime}$ as the dual space of $V$, consisting of linear forms which are bounded with respect to the norm \|\|$_{1,2}$.

Following [7, p. 269/270, 305/306], we choose $k(\Omega) \in \mathbb{N}, \alpha(\Omega) \in(0, \infty)$, orthonormal matrices $A_{1}^{(\Omega)}, \ldots, A_{k(\Omega)}^{(\Omega)} \in \mathbb{R}^{3 \times 3}$, vectors $C_{1}^{(\Omega)}, \ldots, C_{k(\Omega)}^{(\Omega)} \in \mathbb{R}^{3}$, and Lipschitz continuous functions $a_{1}^{(\Omega)}, \ldots, a_{k(\Omega)}^{(\Omega)}:[-\alpha(\Omega), \alpha(\Omega)]^{2} \mapsto \mathbb{R}$ such that the following properties hold true: Defining the sets $\Delta^{\gamma}, \Lambda_{i}^{\gamma}, U_{i}^{\gamma}$ by

$$
\begin{aligned}
\Delta^{\gamma} & :=(-\gamma \cdot \alpha(\Omega), \gamma \cdot \alpha(\Omega))^{2}, \quad \Lambda_{i}^{\gamma}:=\left\{A_{i}^{(\Omega)} \cdot\left(\eta, a_{i}^{(\Omega)}(\eta)\right)+C_{i}^{(\Omega)}: \eta \in \Delta^{\gamma}\right\}, \\
U_{i}^{\gamma} & :=\left\{A_{i}^{(\Omega)} \cdot\left(\eta, a_{i}^{(\Omega)}(\eta)+r\right)+C_{i}^{(\Omega)}: \eta \in \Delta^{\gamma}, \quad r \in(-\gamma \cdot \alpha(\Omega), \gamma \cdot \alpha(\Omega))\right\}
\end{aligned}
$$

for $i \in\{1, \ldots, k(\Omega)\}, \gamma \in(0,1]$, and the function $H^{(i)}: \Delta^{1} \times(-\alpha(\Omega), \alpha(\Omega)) \mapsto U_{i}^{1}$ by

$$
H^{(i)}(\eta, r):=A_{i}^{(\Omega)} \cdot\left(\eta, a_{i}^{(\Omega)}(\eta)+r\right)+C_{i}^{(\Omega)} \quad \text { for } \eta \in \Delta^{1}, r \in(-\alpha(\Omega), \alpha(\Omega)),
$$

we have

$$
\begin{gathered}
U_{i}^{1} \cap \bar{\Omega}^{c}=H^{(i)}\left(\Delta^{1} \times(-\alpha(\Omega), 0)\right), \quad U_{i}^{1} \cap \Omega=H^{(i)}\left(\Delta^{1} \times(0, \alpha(\Omega))\right), \\
U_{i}^{1} \cap \partial \Omega:=\Lambda_{i}^{1} \text { for } i \in\{1, \ldots, k(\Omega)\}, \quad \partial \Omega=\bigcup_{i=1}^{k(\Omega)} \Lambda_{i}^{1 / 4} .
\end{gathered}
$$

These relations imply that

$$
\int_{U_{i}^{1}} g(x) d x=\int_{-\alpha(\Omega)}^{\alpha(\Omega)} \int_{\Delta^{1}}\left(g \circ H^{(i)}\right)(\eta, s) d \eta d s \quad \text { for } g \in L^{1}\left(U_{i}^{1}\right),
$$

and that there is a constant $\mathcal{D}_{1}>0$ with

$$
\begin{gathered}
\left|H^{(i)}(\rho, \kappa)-H^{(i)}\left(\eta, \kappa^{\prime}\right)\right| \geq \mathcal{D}_{1} \cdot\left(|\rho-\eta|+\left|\kappa-\kappa^{\prime}\right|\right) \\
\text { for } \rho, \eta \in \Delta^{1}, \quad \kappa, \kappa^{\prime} \in(-\alpha(\Omega), \alpha(\Omega)), \quad i \in\{1, \ldots, k(\Omega)\} .
\end{gathered}
$$

We further introduce functions $h^{(i)}: \Delta^{1} \mapsto \Lambda_{i}^{1}, \quad J_{i}: \Delta^{1} \mapsto \mathbb{R}$ by setting

$$
h^{(i)}(\eta):=A_{i}^{(\Omega)} \cdot\left(\eta, a_{i}^{(\Omega)}(\eta)\right)+C_{i}^{(\Omega)}, \quad J^{(i)}(\eta):=\left(1+\sum_{r=1}^{2}\left|\partial_{r} h^{(i)}(\eta)\right|^{2}\right)^{1 / 2}
$$

for $\eta \in \Delta^{1}, i \in\{1, \ldots, k(\Omega)\}$. Then we have for any integrable function $g: \partial \Omega \mapsto \mathbb{R}$ and for $i \in\{1, \ldots, k(\Omega)\}$ :

$$
\int_{\Lambda_{i}^{1}} g d \Omega=\int_{\Delta^{1}}\left(g \circ h^{(i)}\right)(\eta) \cdot J^{(i)}(\eta) d \eta
$$

Moreover, let $m^{(\Omega)} \in C_{0}^{\infty}\left(\mathbb{R}^{3}\right)^{3}$ be a non-tangential vector field to $\Omega$. This means that $\left|m^{(\Omega)}(x)\right|=1$ for $x$ from a neighbourhood of $\partial \Omega$ in $\mathbb{R}^{3}$, and there are constants $\mathcal{D}_{2}, \mathcal{D}_{3} \in$ $(0, \infty)$ such that

$$
\left|x+\delta \cdot m^{(\Omega)}(x)-x^{\prime}-\delta^{\prime} \cdot m^{(\Omega)}\left(x^{\prime}\right)\right| \geq \mathcal{D}_{2} \cdot\left(\left|x-x^{\prime}\right|+|\delta-\delta|^{\prime} \mid\right)
$$

for $x, x^{\prime} \in \partial \Omega, \delta, \delta^{\prime} \in\left[-\mathcal{D}_{3}, \mathcal{D}_{3}\right]$, and

$$
x+\delta \cdot m^{(\Omega)}(x) \in \Omega, \quad x-\delta \cdot m^{(\Omega)}(x) \in \bar{\Omega}^{c} \quad \text { for } x \in \partial \Omega, \delta \in\left(0, \mathcal{D}_{3}\right] .
$$


Some indications on how to construct such a field are given in $[9$, p. 246]. Note that since $\Omega$ is only Lipschitz bounded, the relations in (9) and (10) do not hold in general when $m^{(\Omega)}$ is replaced by the outward unit normal to $\Omega$. We further observe that

$$
\operatorname{dist}\left(U_{i}^{1 / 4}, \partial \Omega \backslash \Lambda_{i}^{1 / 2}\right)>0 \text { for } i \in\{1, \ldots, k(\Omega)\}, \text { and } \operatorname{dist}\left(\partial \Omega, \mathbb{R}^{3} \backslash \cup_{i=1}^{k(\Omega)} U_{i}^{1 / 4}\right)>0 .
$$

Thus there is a constant $\mathcal{D}_{4}>0$ such that

$$
\begin{gathered}
|x-y| \geq \mathcal{D}_{4} \text { for } y \in \partial \Omega, \quad x \in \Omega_{R_{0}} \backslash \cup_{i=1}^{k(\Omega)}\left(U_{i}^{1 / 4} \cap \Omega\right), \\
\text { and for } y \in \partial \Omega \backslash \Lambda_{i}^{1 / 2}, \quad x \in U_{i}^{1 / 4}, \quad i \in\{1, \ldots, k(\Omega)\} .
\end{gathered}
$$

We write $\mathcal{C}$ for constants which only depend on $\operatorname{diam} \Omega, \alpha(\Omega), k(\Omega), \mathcal{D}_{1}, \ldots, \mathcal{D}_{4}$, on an upper bound $\mathcal{D}_{5}$ of $\left|a_{i}^{(\Omega)}(\eta)\right|$, with $\eta \in \Delta^{1}$ and $i \in\{1, \ldots, k(\Omega)\}$, and on the Reynolds number $\tau$ from (1). This latter number will be kept fixed throughout. If $n \in \mathbb{N}$ and if $q_{1}, \ldots, q_{n} \in(0, \infty)$ are some other parameters, we write $\mathcal{C}\left(q_{1}, \ldots, q_{n}\right)$ for constants depending on $q_{1}, \ldots, q_{n}$ and also on the quantities just mentioned.

Next we state two results which are frequently used in the context of the Oseen system. The first one, for which we refer to [3, Lemma 4.8], reads as follows:

LEMMA 2.1. There is a constant $C(\tau)>0$ such that

$$
\left(1+\tau \cdot\left(|x-y|-(x-y)_{1}\right)\right)^{-1} \leq C(\tau) \cdot(1+|y|) \cdot\left(1+\tau \cdot\left(|x|-x_{1}\right)\right)^{-1}
$$

for $x, y \in \mathbb{R}^{3}$.

The second one is a special case of [6, Lemma 4.3] and is stated here as

Lemma 2.2. Let $\beta \in(1, \infty)$. Then there is $C=C(\tau, \beta)>0$ such that

$$
\int_{\partial B_{r}}\left(1+\tau \cdot\left(|x|-x_{1}\right)\right)^{-\beta} d o_{x} \leq C \cdot r \quad \text { for } r \in(0, \infty) .
$$

Our main tools in the following will be Minkowski's inequality for integrals and Young's inequality for convolutions. For the convenience of the reader, we state these inequalities in the ensuing two theorems, in a form as in [12, p. 271]. As concerns the proof, we refer to [1, p. 26, Theorem 2.9; p. 34, Corollary 2.25].

THEOREM 2.1. (Minkowski's inequality for integrals) Let $p \in[1, \infty), F: X \times Y \mapsto \mathbb{R} a$ measurable function on the $\sigma$-finite product measure space $X \times Y$. Then

$$
\left(\int_{Y}\left(\int_{X}|F(x, y)| d x\right)^{p} d y\right)^{1 / p} \leq \int_{X}\left(\int_{Y}|F(x, y)|^{p} d y\right)^{1 / p} d x
$$

where $d x$ and $d y$ denote integration with respect to the measures of $X$ and $Y$, respectively.

THEOREM 2.2. (Young's inequality for convolutions) Let $n \in \mathbb{N}, p, q, r \in[1, \infty]$ with $1 / q=1 / p+1 / r-1, f \in L^{p}\left(\mathbb{R}^{n}\right), g \in L^{r}\left(\mathbb{R}^{n}\right)$. Then the convolution $f * g$ is well defined and belongs to $L^{q}\left(\mathbb{R}^{n}\right)$, with $\|f * g\|_{q} \leq\|f\|_{p} \cdot\|g\|_{r}$.

Next we introduce some fundamental solutions. Let $\mathcal{H}$ denote the usual heat kernel in $\mathbb{R}^{3}$, that is,

$$
\mathcal{H}(z, t):=(4 \cdot \pi \cdot t)^{-3 / 2} \cdot e^{-|z|^{2} /(4 \cdot t)} \quad \text { for } z \in \mathbb{R}^{3}, t \in(0, \infty) .
$$


We define a fundamental solution of the time-dependent Stokes system by setting

$$
\Gamma_{j k}(z, t):=\delta_{j k} \cdot \mathcal{H}(z, t)+\int_{t}^{\infty} \partial_{z_{j}} \partial_{z_{k}} \mathcal{H}(z, s) d s, \quad E_{k}(x):=(4 \cdot \pi)^{-1} \cdot x_{k} \cdot|x|^{-3}
$$

for $z \in \mathbb{R}^{3}, t \in(0, \infty), x \in \mathbb{R}^{3} \backslash\{0\}, j, k \in\{1,2,3\}$; compare [11]. The functions $\Gamma_{j k}$ constitute the velocity part, and the functions $E_{k}$ the pressure part of this fundamental solution. The following inequality is well known:

Lemma 2.3. There is $C>0$ such that for $\alpha \in \mathbb{N}_{0}^{3}$ with $|\alpha|_{1} \leq 1, z \in \mathbb{R}^{3}, t \in(0, \infty)$, the estimate $\left|\partial_{z}^{\alpha} \Gamma_{j k}(z, t)\right| \leq C \cdot\left(|z|^{2}+t\right)^{-3 / 2-|\alpha|_{1} / 2}$ holds.

Of course, if $m \in \mathbb{N}, m>1$, the same estimate is valid for $\alpha \in \mathbb{N}_{0}^{3}$ with $|\alpha|_{1} \leq m$, with a constant $C$ depending on $m$; derivatives with respect to $t$ may also be taken into account. But for our purposes, it is sufficient to consider spatial derivatives of first order. We further define the velocity part of a fundamental solution of the time-dependent Oseen system by setting

$$
\Lambda_{j k}(z, t, \tau):=\Gamma\left(z-\tau \cdot t \cdot e_{1}, t\right) \quad \text { for } j, k \in\{1,2,3\}, z \in \mathbb{R}^{3}, t \in(0, \infty) .
$$

(The pressure part of this fundamental solution consists of the functions $E_{k}$ introduced above in the Stokes case.) According to [2, Lemma 3], the function $\Lambda_{j k}$ may be estimated as follows:

Lemma 2.4. For any $K \in(0, \infty)$, there is a constant $C=C(\tau, K)>0$ such that

$$
\left|\partial_{z}^{\alpha} \Lambda_{j k}(z, t, \tau)\right| \leq C \cdot \gamma(z, t)^{-3 / 2-|\alpha|_{1} / 2}
$$

for $z \in \mathbb{R}^{3} \backslash\{0\}, t \in(0, \infty), j, k \in\{1,2,3\}, \alpha \in \mathbb{N}_{0}^{3}$ with $|\alpha|_{1} \leq 1$, where $\gamma(z, t):=$ $|z|^{2}+t$ if $|z| \leq K$, and $\gamma(z, t):=|z| \cdot\left(1+\tau \cdot\left(|z|-z_{1}\right)\right)+t$ if $|z|>K$.

Next we introduce our single-layer potentials. For $T \in(0, \infty], \Phi \in L^{2}\left(0, T, L^{1}(\partial \Omega)^{3}\right)$, $z \in \mathbb{R}^{3}, x \in \mathbb{R}^{3} \backslash \partial \Omega, t \in[0, T] \cap \mathbb{R}$, we put

$$
\begin{gathered}
\mathcal{V}_{T}^{(\tau)}(\Phi)(z, t):=\left(\int_{0}^{t} \int_{\partial \Omega} \sum_{k=1}^{3} \Lambda_{j k}(x-y, t-\sigma, \tau) \cdot \Phi_{k}(y, \sigma) d \Omega(y) d \sigma\right)_{1 \leq j \leq 3}, \\
Q_{T}(\Phi)(x, t):=\int_{\partial \Omega} \sum_{k=1}^{3} E_{k}(x-y) \cdot \Phi_{k}(y, t) d \Omega(y) .
\end{gathered}
$$

The pair $\left(\mathcal{V}_{T}^{(\tau)}(\Phi), Q_{T}(\Phi)\right)$ is called the "single-layer potential associated to the timedependent Oseen system", with layer function $\Phi$. According to the following lemma, this pair solves equations (1) and (3) with $f=0, a=0$.

Lemma 2.5. Let $T \in(0, \infty], \Phi \in L^{2}\left(0, T, L^{1}(\partial \Omega)^{3}\right)$, and abbreviate

$$
v:=\mathcal{V}_{T}^{(\tau)}(\Phi) \mid\left(\mathbb{R}^{3} \backslash \partial \Omega\right) \times([0, T] \cap \mathbb{R}), \quad q:=Q_{T}(\Phi) .
$$

Then $v \in C^{0}\left(\left(\mathbb{R}^{3} \backslash \partial \Omega\right) \times([0, T] \cap \mathbb{R})\right)^{3}, \quad v_{j}(\cdot, t), q(\cdot, t) \in C^{\infty}\left(\mathbb{R}^{3} \backslash \partial \Omega\right)$ for $1 \leq j \leq 3$ and for a.e. $t \in(0, T)$, the derivative $\partial_{t} v(x, t)$ exists and

$$
\partial_{t} v(x, t)-\Delta_{x} v(x, t)+\tau \cdot \partial_{x_{1}} v(x, t)+\nabla_{x} q(x, t)=0, \quad \operatorname{div}_{x} v(x, t)=0, \quad v(x, 0)=0
$$

for $x \in \mathbb{R}^{3} \backslash \partial \Omega$ and a.e. $t \in(0, T)$, and $v(x, t) \rightarrow 0(|x| \rightarrow \infty)$ for $t \in[0, T] \cap \mathbb{R}$. 
This lemma follows from Lebesgue's theorem on dominated convergence and from the equations

$$
\partial_{t} \Lambda_{j k}(x, t)-\Delta_{x} \Lambda_{j k}(x, t)+\tau \cdot \partial_{x_{1}} \Lambda_{j k}(x, t)=0, \quad \sum_{i=1}^{3} \partial_{x_{i}} \Lambda_{i k}(x, t)=0
$$

for $x \in \mathbb{R}^{3}, t \in(0, \infty), 1 \leq j, k \leq 3$. Now we may state the main result of the present article.

Theorem 2.3. Let $p \in(4 / 3,2], T \in(0, \infty]$. Then the function $\partial_{t}\left(\mathcal{V}_{T}^{(\tau)}(\Phi) \mid Z_{T}\right)$ may be considered as a mapping from $(0, T)$ into $V^{\prime}$, for any $\Phi \in L^{2}\left(0, T, L^{p}(\partial \Omega)^{3}\right)$, if this mapping is defined by

$$
\partial_{t}\left(\mathcal{V}_{T}^{(\tau)}(\Phi) \mid Z_{T}\right)(t)(w)=\int_{\Omega} \partial_{t} \mathcal{V}_{T}^{(\tau)}(\Phi)(x, t) \cdot w(x) d x
$$

for $w \in C_{0}^{\infty}(\Omega)^{3}$ with div $w=0$ and for a.e. $t \in(0, T)$. There is a constant $C>0$, depending on $\tau$, diam $\Omega, k(\Omega), \alpha(\Omega), \mathcal{D}_{1}, \ldots, \mathcal{D}_{5}$ and $p$, such that the inequality

$$
\begin{aligned}
& \left\|\mathcal{V}_{T}^{(\tau)}(\Phi) \mid Z_{T}\right\|_{L^{\infty}\left(0, T, L^{2}(\Omega)^{3}\right)}+\left\|\nabla\left(\mathcal{V}_{T}^{(\tau)}(\Phi) \mid Z_{T}\right)\right\|_{2} \\
& +\left\|\partial_{t}\left(\mathcal{V}_{T}^{(\tau)}(\Phi) \mid Z_{T}\right)\right\|_{L^{2}\left(0, T, V^{\prime}\right)} \leq C \cdot\|\Phi\|_{L^{2}\left(0, T, L^{p}(\partial \Omega)^{3}\right)}
\end{aligned}
$$

holds for $T \in(0, \infty], \Phi \in L^{2}\left(0, T, L^{p}(\partial \Omega)^{3}\right)$.

We will also show the following

Theorem 2.4. Let $p \in(4 / 3,2], T \in(0, \infty], \Phi \in L^{2}\left(0, T, L^{p}(\partial \Omega)^{3}\right)$. Then, for a.e. $t \in(0, T)$, the trace of $\mathcal{V}_{T}^{(\tau)}(\Phi)(\cdot, t) \mid \Omega$ on $\partial \Omega$ coincides with $\mathcal{V}_{T}^{(\tau)}(\Phi)(\cdot, t) \mid \partial \Omega$, that is,

$$
\operatorname{trace}\left(\mathcal{V}_{T}^{(\tau)}(\Phi)(\cdot, t) \mid \Omega\right)_{j}(x)=\int_{0}^{t} \int_{\partial \Omega} \sum_{k=1}^{3} \Lambda_{j k}(x-y, t-\sigma, \tau) \cdot \Phi_{k}(y, \sigma) d \Omega(y) d \sigma
$$

for $j \in\{1,2,3\}$ and for a.e. $t \in(0, T), x \in \partial \Omega$.

Let us still state a consequence of Theorem 2.3.

Corollary 2.1. Let $p, T, \Phi$ be given as in Theorem 2.4. Then the function $\mathcal{V}_{T}^{(\tau)}(\Phi) \mid Z_{T}$ may be considered as a mapping from $(0, T)$ into $V^{\prime}$ in the sense that

$$
\left(\mathcal{V}_{T}^{(\tau)}(\Phi) \mid Z_{T}\right)(t)(w)=\int_{\Omega} \mathcal{V}_{T}^{(\tau)}(\Phi)(x, t) \cdot w(x) d x \quad \text { for } w \in V, t \in(0, T) .
$$

Let $\partial_{t}\left(\mathcal{V}_{T}^{(\tau)}(\Phi) \mid Z_{T}\right)$ be understood as a mapping from $(0, T)$ into $V^{\prime}$ as in Theorem 2.3. Then $\mathcal{V}_{T}^{(\tau)}(\Phi) \mid Z_{T} \in L^{2}(0, T, V) \cap H^{1}\left(0, T, V^{\prime}\right)$, with $\left(\mathcal{V}_{T}^{(\tau)}(\Phi) \mid Z_{T}\right)^{\prime}=\partial_{t}\left(\mathcal{V}_{T}^{(\tau)}(\Phi) \mid Z_{T}\right)$.

3. Proof of Theorem 2.3 and 2.4. For $T \in(0, \infty), p \in[1,2]$ and for any function $\Phi \in L^{2}\left(0, T, L^{p}(\partial \Omega)^{3}\right)$ (whose domain is $S_{T}$ ), the zero extension of $\Phi$ to $S_{\infty}$ belongs to $L^{2}\left(0, \infty, L^{p}(\partial \Omega)^{3}\right)$. Moreover, for $p, T$ as before, and for $\Phi \in L^{2}\left(0, \infty, L^{p}(\partial \Omega)^{3}\right)$, we have

$$
\mathcal{V}_{\infty}^{(\tau)}(\Phi)(x, t)=\mathcal{V}_{T}^{(\tau)}\left(\Phi \mid S_{T}\right)(x, t) \quad \text { for }(x, t) \in \overline{Z_{T}}
$$

Thus, without loss of generality, we may restrict ourselves to the case $T=\infty$.

The key estimates leading to Theorem 2.3 and 2.4 are given by the two ensuing lemmas. 
Lemma 3.1. For $\Phi \in L^{2}\left(0, \infty, L^{1}(\partial \Omega)^{3}\right), t \in[0, \infty), x \in \Omega_{R_{0}}, \nu \in\{0,1\}$, we put

$$
\mathcal{K}_{\nu}(\Phi)(x, t):=\int_{0}^{t} \int_{\partial \Omega}\left(|x-y|^{2}+t-\sigma\right)^{-3 / 2-\nu / 2} \cdot|\Phi(y, \sigma)| d \Omega(y) d \sigma .
$$

Then, for $p \in(4 / 3,2], \Phi \in L^{2}\left(0, \infty, L^{p}(\partial \Omega)^{3}\right), t \in[0, \infty)$, the following inequality is valid:

$$
\left\|\mathcal{K}_{0}(\Phi)(\cdot, t)\right\|_{2}+\left\|\mathcal{K}_{1}(\Phi)\right\|_{2} \leq \mathcal{C}(p) \cdot\|\Phi\|_{L^{2}\left(0, \infty, L^{p}(\partial \Omega)^{3}\right)} \cdot
$$

Proof. Since $\|v\|_{q} \leq \mathcal{C} \cdot\|v\|_{2}$ for $q \in[1,2), v \in L^{2}(\partial \Omega)$, we may assume without loss of generality that $p<2$. Thus let $p \in(4 / 3,2)$. Take $\Phi$ as in the lemma, and let $\nu \in\{0,1\}$. Then, for $t \in(0, \infty)$,

$$
\int_{\Omega_{R_{0}}} \mathcal{K}_{\nu}(\Phi)(x, t)^{2} d x \leq A(t)+2 \cdot \sum_{i=1}^{k(\Omega)}\left(B_{i}(t)+C_{i}(t)\right),
$$

with

$$
\begin{array}{r}
A(t):=\int_{M}\left(\int_{0}^{t} \int_{\partial \Omega}\left(|x-y|^{2}+t-\sigma\right)^{-3 / 2-\nu / 2} \cdot|\Phi(y, \sigma)| d \Omega(y) d \sigma\right)^{2} d x \\
B_{i}(t):=\int_{U_{i}^{1 / 4} \cap \Omega}\left(\int_{0}^{t} \int_{\partial \Omega \backslash \Lambda_{i}^{1 / 2}}\left(|x-y|^{2}+t-\sigma\right)^{-3 / 2-\nu / 2} \cdot|\Phi(y, \sigma)| d \Omega(y) d \sigma\right)^{2} d x \\
C_{i}(t):=\int_{U_{i}^{1 / 4} \cap \Omega}\left(\int_{0}^{t} \int_{\Lambda_{i}^{1 / 2}}\left(|x-y|^{2}+t-\sigma\right)^{-3 / 2-\nu / 2} \cdot|\Phi(y, \sigma)| d \Omega(y) d \sigma\right)^{2} d x
\end{array}
$$

for $i \in\{1, \ldots, k(\Omega)\}$, where $M:=\Omega_{R_{0}} \backslash \cup_{i=1}^{k(\Omega)}\left(U_{i}^{1 / 4} \cap \Omega\right)$. By (11) and Hölder's inequality, we get for $t \in(0, \infty)$,

$$
\begin{gathered}
A(t)+\sum_{i=1}^{k(\Omega)} B_{i}(t) \leq \mathcal{C} \cdot \int_{\Omega_{R_{0}}}\left(\int_{0}^{t} \int_{\partial \Omega}\left(\mathcal{D}_{4}^{2}+t-\sigma\right)^{-3 / 2-\nu / 2} \cdot|\Phi(y, \sigma)| d \Omega(y) d \sigma\right)^{2} d x \\
\leq \mathcal{C} \cdot\left(\int_{0}^{t}\left(\mathcal{D}_{4}^{2}+t-\sigma\right)^{-3 / 2-\nu / 2} \cdot\|\Phi(\cdot, \sigma)\|_{p} d \sigma\right)^{2} .
\end{gathered}
$$

Therefore, in the case $\nu=0$, by Hölder's inequality,

$$
\begin{aligned}
A(t)+\sum_{i=1}^{k(\Omega)} B_{i}(t) \leq & \mathcal{C} \cdot\left(\int_{0}^{t}\left(\mathcal{D}_{4}^{2}+t-\sigma\right)^{-3} d \sigma\right) \cdot\|\Phi\|_{L^{2}\left(0, \infty, L^{p}(\partial \Omega)^{3}\right)}^{2} \\
& \leq \mathcal{C} \cdot\|\Phi\|_{L^{2}\left(0, \infty, L^{p}(\partial \Omega)^{3}\right)}^{2} \quad(t \in(0, \infty)) .
\end{aligned}
$$

In the case $\nu=1$, we deduce from (13), using Young's inequality (Theorem 2.2),

$$
\begin{aligned}
\int_{0}^{\infty}\left(A(t)+\sum_{i=1}^{k(\Omega)} B_{i}(t)\right) d t & \leq \mathcal{C} \cdot\left(\int_{0}^{\infty}\left(\mathcal{D}_{4}^{2}+s\right)^{-2} d s\right)^{2} \cdot\|\Phi\|_{L^{2}\left(0, \infty, L^{p}(\partial \Omega)^{3}\right)}^{2} \\
& \leq \mathcal{C} \cdot\|\Phi\|_{L^{2}\left(0, \infty, L^{p}(\partial \Omega)^{3}\right)}^{2} .
\end{aligned}
$$

Now take $i \in\{1, \ldots, k(\Omega)\}$ and consider $C_{i}(t)$. Abbreviate

$$
\widetilde{\Phi}^{(i)}(\eta, \sigma):=\Phi\left(h^{(i)}(\eta), \sigma\right) \text { for } \eta \in \Delta^{1 / 2}, \sigma \in(0, \infty) .
$$


Then we get by changes of variables as in (6) and (8), and by referring to (7):

$$
\begin{aligned}
C_{i}(t)= & \int_{0}^{\alpha(\Omega) / 4} \int_{\Delta^{1 / 4}}\left[\int_{0}^{t} \int_{\Delta^{1 / 2}}\left(\left|H^{(i)}(\varrho, r)-h^{(i)}(\eta)\right|^{2}+t-\sigma\right)^{-3 / 2-\nu / 2}\right. \\
\left.\cdot\left|\widetilde{\Phi}^{(i)}(\eta, \sigma)\right| \cdot J_{i}(\eta) d \eta d \sigma\right]^{2} d \varrho d r & \int_{\Delta^{1 / 4}}\left[\int_{0}^{t} \int_{\Delta^{1 / 2}}\left(|\varrho-\eta|+r+(t-\sigma)^{1 / 2}\right)^{-3-\nu}\right. \\
& \left.\leq \mathcal{C} \cdot \int_{0}^{\alpha(\Omega) / 4} \cdot\left|\widetilde{\Phi}^{(i)}(\eta, \sigma)\right| d \eta d \sigma\right]^{2} d \varrho d r \quad(t \in(0, \infty)) .
\end{aligned}
$$

By first applying Minkowski's and then Young's inequality (Theorem 2.1 and 2.2, respectively), we may conclude for $t \in(0, \infty)$ :

$$
\begin{gathered}
C_{i}(t) \leq \mathcal{C} \cdot \int_{0}^{\alpha(\Omega) / 4}\left[\int _ { 0 } ^ { t } \left(\int _ { \Delta ^ { 1 / 4 } } \left(\int_{\Delta^{1 / 2}}\left(|\varrho-\eta|+r+(t-\sigma)^{1 / 2}\right)^{-3-\nu}\right.\right.\right. \\
\left.\left.\left.\cdot\left|\widetilde{\Phi}^{(i)}(\eta, \sigma)\right| d \eta\right)^{2} d \varrho\right)^{1 / 2} d \sigma\right]^{2} d r \\
\leq \mathcal{C} \cdot \int_{0}^{\alpha(\Omega) / 4}\left[\int_{0}^{t}\left(\int_{\mathbb{R}^{2}}\left(|\zeta|+r+(t-\sigma)^{1 / 2}\right)^{(-3-\nu) \cdot(3 / 2-1 / p)^{-1}} d \zeta\right)^{3 / 2-1 / p}\right. \\
\left.\cdot\left\|\widetilde{\Phi}^{(i)}(\cdot, \sigma)\right\|_{p} d \sigma\right]^{2} d r .
\end{gathered}
$$

But $(3+\nu) \cdot(3 / 2-1 / p)^{-1} \geq 3 \cdot(3 / 2-1 / p)^{-1}>2$, so that

$$
\begin{aligned}
& \left(\int_{\mathbb{R}^{2}}\left(|\zeta|+r+(t-\sigma)^{1 / 2}\right)^{(-3-\nu) \cdot(3 / 2-1 / p)^{-1}} d \zeta\right)^{3 / 2-1 / p} \\
& \quad \leq \mathcal{C}(p) \cdot\left(r+(t-\sigma)^{1 / 2}\right)^{-\nu-2 / p} \leq \mathcal{C}(p) \cdot\left(r^{2}+t-\sigma\right)^{-\nu / 2-1 / p}
\end{aligned}
$$

for $r, t, \sigma \in(0, \infty)$ with $\sigma<t$. It follows from (16) that

$$
C_{i}(t) \leq \mathcal{C}(p) \cdot \int_{0}^{\alpha(\Omega) / 4}\left[\int_{0}^{t}\left(r^{2}+t-\sigma\right)^{-\nu / 2-1 / p} \cdot\left\|\widetilde{\Phi}^{(i)}(\cdot, \sigma)\right\|_{p} d \sigma\right]^{2} d r,
$$

for $t \in(0, \infty)$. In the case $\nu=0$, the right-hand side of (17) is dominated by

$$
\begin{aligned}
\mathcal{C}(p) \cdot \int_{0}^{\alpha(\Omega) / 4} & \left(\int_{0}^{t}\left(r^{2}+t-\sigma\right)^{-2 / p} d \sigma\right) d r \cdot\left\|\widetilde{\Phi}^{(i)}\right\|_{L^{2}\left(0, \infty, L^{p}\left(\Delta^{1 / 2}\right)^{3}\right)}^{2} \\
\leq & \mathcal{C}(p) \cdot \int_{0}^{\alpha(\Omega) / 4}\left(r^{2}\right)^{-2 / p+1} d r \cdot\|\Phi\|_{L^{2}\left(0, \infty, L^{p}(\partial \Omega)^{3}\right)}^{2} \quad(t \in(0, \infty)) .
\end{aligned}
$$

In the last inequality, we used that $p<2$, hence $-2 / p<-1$. But $p>4 / 3$, hence $-4 / p+2>-1$, so we may conclude that

$$
C_{i}(t) \leq \mathcal{C}(p) \cdot\|\Phi\|_{L^{2}\left(0, \infty, L^{p}(\partial \Omega)^{3}\right)}^{2} \quad \text { for } t \in(0, \infty) \quad \text { if } \nu=0 .
$$


In the case $\nu=1$, we find with (17) and Young's inequality,

$$
\begin{aligned}
\int_{0}^{\infty} C_{i}(t) d t & \leq \mathcal{C}(p) \cdot \int_{0}^{\infty} \int_{0}^{\alpha(\Omega) / 4}\left[\int_{0}^{t}\left(r^{2}+t-\sigma\right)^{-1 / 2-1 / p} \cdot\left\|\widetilde{\Phi}^{(i)}(\cdot, \sigma)\right\|_{p} d \sigma\right]^{2} d r d t \\
& \leq \mathcal{C}(p) \cdot \int_{0}^{\alpha(\Omega) / 4}\left(\int_{0}^{\infty}\left(r^{2}+s\right)^{-1 / 2-1 / p} d s \cdot\left\|\widetilde{\Phi}^{(i)}\right\|_{L^{2}\left(0, \infty, L^{p}\left(\Delta^{1 / 2}\right)^{3}\right)}^{2}\right)^{2} d r \\
& \leq \mathcal{C}(p) \cdot\left(\int_{0}^{\alpha(\Omega) / 4} r^{1-2 / p} d r\right) \cdot\|\Phi\|_{L^{2}\left(0, \infty, L^{p}(\partial \Omega)^{3}\right)}^{2} \\
& \leq \mathcal{C}(p) \cdot\|\Phi\|_{L^{2}\left(0, \infty, L^{p}(\partial \Omega)^{3}\right)}^{2} .
\end{aligned}
$$

Note that $-1 / 2-1 / p<-1$ because of the assumption $p<2$. In the case $\nu=0$, we may deduce from (12), (14) and (18) that

$$
\int_{\Omega_{R_{0}}} \mathcal{K}_{0}(\Phi)(x, t)^{2} d x \leq \mathcal{C}(p) \cdot\|\Phi\|_{L^{2}\left(0, \infty, L^{p}(\partial \Omega)^{3}\right)}^{2} \quad \text { for } t \in(0, \infty) .
$$

As concerns the case $\nu=1$, we refer to (12), (15) and (19) to obtain

$$
\int_{0}^{\infty} \int_{\Omega_{R_{0}}} \mathcal{K}_{1}(\Phi)(x, t)^{2} d x d t \leq \mathcal{C}(p) \cdot\|\Phi\|_{L^{2}\left(0, \infty, L^{p}(\partial \Omega)^{3}\right)^{2}}^{2} .
$$

Inequality (20) and (21) yield the lemma.

LEMMA 3.2. The inequality

$$
\left\|\mathcal{V}_{\infty}^{(\tau)}(\Phi)(\cdot, t) \mid B_{R_{0}}^{c}\right\|_{2}+\left\|\nabla\left(\mathcal{V}_{\infty}^{(\tau)}(\Phi) \mid B_{R_{0}}^{c} \times(0, \infty)\right)\right\|_{2} \leq \mathcal{C} \cdot\|\Phi\|_{L^{2}\left(0, \infty, L^{p}(\partial \Omega)^{3}\right)}
$$

holds for $p \in[1,2], \Phi \in L^{2}\left(0, \infty, L^{p}(\partial \Omega)^{3}\right), t \in[0, \infty)$.

Proof. Take $p$ and $\Phi$ as in the lemma. Let $j \in\{1,2,3\}, \alpha \in \mathbb{N}_{0}^{3}$ with $|\alpha|_{1} \leq 1$. Then, for $t \in(0, \infty)$

$$
\int_{B_{R_{0}}^{c}}\left|\partial_{x}^{\alpha} \mathcal{V}_{\infty}^{(\tau)}(\Phi)_{j}(x, t)\right|^{2} d x \leq A_{\alpha}(t)+B_{\alpha}(t)
$$

with $A_{\alpha}(t)$ defined as an abbreviation of the term

$$
\int_{B_{R_{0}}^{c}}\left(\int_{0}^{t} \int_{\partial \Omega} \chi_{(0,1)}(t-\sigma) \cdot \sum_{k=1}^{3}\left|\partial_{x}^{\alpha} \Lambda_{j k}(x-y, t-\sigma, \tau)\right| \cdot|\Phi(y, \sigma)| d \Omega(y) d \sigma\right)^{2} d x
$$

and with $B_{\alpha}(t)$ defined in the same way, except that the term $\chi_{(0,1)}(t-\sigma)$ is replaced by $\chi_{(1, \infty)}(t-\sigma)$. Since $\Omega^{c} \subset B_{R_{0} / 2}$, we have $|x-y| \geq|x| / 2 \geq R_{0} / 2$ for $x \in B_{R_{0}}^{c}, y \in \partial \Omega$, so we may conclude from Lemma 2.4 with $K=R_{0} / 2$, and from Lemma 2.1,

$$
\begin{aligned}
\left|\partial_{x}^{\alpha} \Lambda_{j k}(x-y, t-\sigma, \tau)\right| & \leq \mathcal{C} \cdot\left(|x-y| \cdot\left(1+\tau \cdot\left(|x-y|-(x-y)_{1}\right)\right)+t-\sigma\right)^{-3 / 2-|\alpha|_{1} / 2} \\
& \leq \mathcal{C} \cdot\left(|x| \cdot\left(1+\tau \cdot\left(|x|-x_{1}\right)\right)+t-\sigma\right)^{-3 / 2-|\alpha|_{1} / 2}
\end{aligned}
$$

for $x \in B_{R_{0}}^{c}, y \in \partial \Omega, 1 \leq j, k \leq 3, \alpha \in \mathbb{N}_{0}^{3}$ with $|\alpha|_{1} \leq 1, t, \sigma \in(0, \infty)$ with $\sigma<t$. It follows with Lemma 2.2 that

$$
\begin{aligned}
A_{\alpha}(t) \leq \mathcal{C} \cdot \int_{B_{R_{0}}^{c}}(|x| \cdot(1+\tau & \left.\left.\cdot\left(|x|-x_{1}\right)\right)\right)^{-3-|\alpha|_{1}} d x \\
& \cdot\left(\int_{0}^{t} \int_{\partial \Omega} \chi_{(0,1)}(t-\sigma) \cdot|\Phi(y, \sigma)| d \Omega(y) d \sigma\right)^{2}
\end{aligned}
$$




$$
\leq \mathcal{C} \cdot\left(\int_{0}^{t} \chi_{(0,1)}(t-\sigma) \cdot\|\Phi(\cdot, \sigma)\|_{p} d \sigma\right)^{2} .
$$

Now Hölder's inequality yields

$$
A_{0}(t) \leq \mathcal{C} \cdot\|\Phi\|_{L^{2}\left(0, \infty, L^{p}(\partial \Omega)^{3}\right)}^{2} \quad \text { for } t \in(0, \infty),
$$

and Young's inequality (Theorem 2.2) implies for $\alpha \in \mathbb{N}_{0}^{3}$ with $|\alpha|_{1}=1$,

$$
\begin{aligned}
\int_{0}^{\infty} & A_{\alpha}(t) d t \leq \mathcal{C} \cdot \int_{0}^{\infty}\left(\int_{0}^{t} \chi_{(0,1)}(t-\sigma) \cdot\|\Phi(\cdot, \sigma)\|_{p} d \sigma\right)^{2} d t \\
& \leq \mathcal{C} \cdot\left(\int_{0}^{\infty} \chi_{(0,1)}(s) d s\right)^{2} \cdot\|\Phi\|_{L^{2}\left(0, \infty, L^{p}(\partial \Omega)^{3}\right)}^{2} \leq \mathcal{C} \cdot\|\Phi\|_{L^{2}\left(0, \infty, L^{p}(\partial \Omega)^{3}\right)^{2}}^{2}
\end{aligned}
$$

Concerning $B(t)$, we obtain by Minkowski's inequality (Theorem 2.1),

$$
\begin{array}{r}
B_{\alpha}(t) \leq \mathcal{C} \cdot \sum_{k=1}^{3}\left(\int_{0}^{t} \int_{\partial \Omega} \chi_{(1, \infty)}(t-\sigma) \cdot\left(\int_{\mathbb{R}^{3}}\left|\partial_{x}^{\alpha} \Lambda_{j k}(x-y, t-\sigma, \tau)\right|^{2} d x\right)^{1 / 2}\right. \\
\cdot|\Phi(y, \sigma)| d \Omega(y) d \sigma)^{2} \quad\left(t \in(0, \infty), \alpha \in \mathbb{N}_{0}^{3},|\alpha|_{1} \leq 1\right) .
\end{array}
$$

But

$$
\begin{aligned}
& \int_{\mathbb{R}^{3}}\left|\partial_{x}^{\alpha} \Lambda_{j k}(x-y, t-\sigma, \tau)\right|^{2} d y=\int_{\mathbb{R}^{3}}\left|\partial_{z}^{\alpha} \Gamma_{j k}(z, t-\sigma)\right|^{2} d z \\
\leq & \mathcal{C} \cdot \int_{\mathbb{R}^{3}}\left(|z|+(t-\sigma)^{1 / 2}\right)^{-6-2 \cdot|\alpha|_{1}} d z \leq \mathcal{C} \cdot(t-\sigma)^{-3 / 2-|\alpha|_{1}}
\end{aligned}
$$

$\left(x \in \mathbb{R}^{3}, t, \sigma \in(0, \infty)\right.$ with $\left.t>\sigma, 1 \leq j, k \leq 3\right)$; see Lemma 2.3. Thus we may deduce from (25), by Hölder's inequality,

$$
\begin{aligned}
B_{0}(t) & \leq \mathcal{C} \cdot\left(\int_{0}^{t} \int_{\partial \Omega} \chi_{(1, \infty)}(t-\sigma) \cdot(t-\sigma)^{-3 / 4} \cdot|\Phi(y, \sigma)| d \Omega(y) d \sigma\right)^{2} \\
& \leq \mathcal{C} \cdot\|\Phi\|_{L^{2}\left(0, \infty, L^{p}(\partial \Omega)^{3}\right)}^{2} \quad(t \in(0, \infty)),
\end{aligned}
$$

and by Hölder's and Young's inequality (Theorem 2.2), if $\alpha \in \mathbb{N}_{0}^{3}$ with $|\alpha|=1$ :

$$
\begin{aligned}
& \int_{0}^{\infty} B_{\alpha}(t) d t \leq \int_{0}^{\infty}\left(\int_{0}^{t} \int_{\partial \Omega} \chi_{(1, \infty)}(t-\sigma) \cdot(t-\sigma)^{-5 / 4} \cdot|\Phi(y, \sigma)| d \Omega(y) d \sigma\right)^{2} d t \\
& \leq \mathcal{C} \cdot \int_{0}^{\infty}\left(\int_{0}^{t} \chi_{(1, \infty)}(t-\sigma) \cdot(t-\sigma)^{-5 / 4} \cdot\|\Phi(\cdot, \sigma)\|_{p} d \sigma\right)^{2} d t \\
& \leq \mathcal{C} \cdot\left(\int_{\mathbb{R}} \chi_{(1, \infty)}(r) \cdot r^{-5 / 4} d r\right)^{2} \cdot\|\Phi\|_{L^{2}\left(0, \infty, L^{p}(\partial \Omega)^{3}\right)}^{2} \leq \mathcal{C} \cdot\|\Phi\|_{L^{2}\left(0, \infty, L^{p}(\partial \Omega)^{3}\right)^{2}}^{2}
\end{aligned}
$$

The lemma follows from (22) - (24), (26), (27).

As a first consequence of Lemma 3.1 and 3.2, we obtain an estimate of $\mathcal{V}_{\infty}^{(\tau)}(\Phi) \mid Z_{\infty}$ and $\nabla_{x}\left(\mathcal{V}_{\infty}^{(\tau)}(\Phi) \mid Z_{\infty}\right)$ :

Corollary 3.1. For $p \in(4 / 3,2], \Phi \in L^{2}\left(0, \infty, L^{p}(\partial \Omega)^{3}\right), t \in[0, \infty)$, the inequality

$$
\left\|\mathcal{V}_{\infty}^{(\tau)}(\Phi)(\cdot, t) \mid \Omega\right\|_{2}+\left\|\nabla_{x}\left(\mathcal{V}_{\infty}^{(\tau)}(\Phi) \mid Z_{\infty}\right)\right\|_{2} \leq \mathcal{C}(p) \cdot\|\Phi\|_{L^{2}\left(0, \infty, L^{p}(\partial \Omega)^{3}\right)}
$$

holds. 
Proof. Since $|x-y| \leq 2 \cdot R_{0}$ for $x \in \Omega_{R_{0}}$ and $y \in \partial \Omega$, Lemma 2.4 with $K=2 \cdot R_{0}$ shows that $\left|\partial_{x}^{\alpha} \Lambda_{j k}(x-y, t-\sigma, \tau)\right|$ is dominated by $\mathcal{C} \cdot\left(|x-y|^{2}+t-\sigma\right)^{-3 / 2-|\alpha|_{1} / 2}$ for $x \in \Omega_{R_{0}}, y \in \partial \Omega, t \in(0, \infty), \sigma \in(0, t), \alpha \in \mathbb{N}_{0}^{3}$ with $|\alpha|_{1} \leq 1$. Thus Corollary 3.1 follows from Lemma 3.1 and 3.2.

We complete the proof of Theorem 2.3 by establishing the following Lemma 3.3. The inequality

$$
\left\|\partial_{t}\left(\mathcal{V}_{\infty}^{(\tau)}(\Phi) \mid Z_{\infty}\right)\right\|_{L^{2}\left(0, \infty, V^{\prime}\right)} \leq \mathcal{C}(p) \cdot\|\Phi\|_{L^{2}\left(0, \infty, L^{p}(\partial \Omega)^{3}\right)}
$$

holds for $p \in(4 / 3,2], \Phi \in L^{2}\left(0, \infty, L^{p}(\partial \Omega)^{3}\right)$.

Proof. Take $p, \Phi$ as in the lemma, and abbreviate $\mathcal{V}:=\mathcal{V}_{\infty}^{(\tau)}(\Phi) \mid Z_{\infty}$. Let $t \in(0, \infty), \varphi \in$ $C_{0}^{\infty}(\Omega)$ with $\operatorname{div} \varphi=0$. Then we get, by Lemma 2.5 ,

$$
\begin{aligned}
\left|\int_{\Omega} \partial_{t} \mathcal{V}(x, t) \cdot \varphi(x) d x\right| & =\left|\int_{\Omega}\left(\Delta_{x} \mathcal{V}(x, t)-\tau \cdot \partial_{x_{1}} \mathcal{V}(x, t)-\nabla_{x} Q(\Phi)(x, t)\right) \cdot \varphi(x) d x\right| \\
& =\left|\int_{\Omega}\left(-\nabla_{x} \mathcal{V}(x, t) \cdot \nabla \varphi(x)-\tau \cdot \partial_{x_{1}} \mathcal{V}(x, t) \cdot \varphi(x)\right) d x\right| \\
& \leq \mathcal{C} \cdot\left(\int_{\Omega}\left|\nabla_{x} \mathcal{V}(x, t)\right|^{2} d x\right)^{1 / 2} \cdot\|\varphi\|_{1,2} .
\end{aligned}
$$

It follows that $\left\|\partial_{t} \mathcal{V}(\cdot, t)\right\|_{V^{\prime}}^{2} \leq \mathcal{C} \cdot \int_{\Omega}\left|\nabla_{x} \mathcal{V}(x, t)\right|^{2} d x$ for $t \in(0, \infty)$, so Lemma 3.3 follows from Corollary 3.1.

Let us now turn to the proof of Theorem 2.4. We introduce two additional notations. For $\kappa \in\left(0, \mathcal{D}_{3}\right]$, we put

$$
\widetilde{\Omega}_{\kappa}:=\left\{x \in \mathbb{R}^{3}: \operatorname{dist}(x, \Omega)<\mathcal{D}_{2} \cdot \kappa / 2\right\} .
$$

Note that $\bar{\Omega} \subset \widetilde{\Omega}_{\kappa}$. For $\Phi \in L^{2}\left(0, \infty, L^{1}(\partial \Omega)^{3}\right), \kappa \in\left(0, \mathcal{D}_{3}\right], x \in \widetilde{\Omega}_{\kappa}, t \in(0, \infty), j \in$ $\{1,2,3\}$, we define

$$
\mathcal{V}^{(\tau, \kappa)}(\Phi)_{j}(x, t):=\int_{0}^{t} \int_{\partial \Omega} \sum_{k=1}^{3} \Lambda_{j k}\left(x-y+\kappa \cdot m^{(\Omega)}(y), t-\sigma, \tau\right) \cdot \Phi_{k}(y, \sigma) d \Omega(y) d \sigma .
$$

Lemma 3.4. For $\kappa, \Phi$ as in (28), we have

$$
\begin{aligned}
& \mathcal{V}^{(\tau, \kappa)}(\Phi) \in C^{0}\left(\widetilde{\Omega}_{\kappa} \times(0, \infty)\right)^{3}, \quad \mathcal{V}^{(\tau, \kappa)}(\Phi)(\cdot, t) \in C^{1}\left(\widetilde{\Omega}_{\kappa}\right)^{3} \quad \text { for } t \in(0, \infty), \\
& \partial_{x}^{\alpha} \mathcal{V}^{(\tau, \kappa)}(\Phi)_{j}(x, t) \\
& \quad=\int_{0}^{t} \int_{\partial \Omega} \sum_{k=1}^{3} \partial_{x}^{\alpha} \Lambda_{j k}\left(x-y+\kappa \cdot m^{(\Omega)}(y), t-\sigma, \tau\right) \cdot \Phi_{k}(y, \sigma) d \Omega(y) d \sigma
\end{aligned}
$$

for $\kappa, x, t, j$ as in (28), $\alpha \in \mathbb{N}_{0}^{3}$ with $|\alpha|_{1} \leq 1$. Moreover,

$$
\left|\partial_{x}^{\alpha} \Lambda_{j k}\left(x-y+\kappa \cdot m^{(\Omega)}(y), t-\sigma, \tau\right)\right| \leq \mathcal{C} \cdot\left(|x-y|^{2}+t-\sigma\right)^{-3 / 2-|\alpha|_{1} / 2}
$$

for $x \in \Omega_{R_{0}}, y \in \partial \Omega, \kappa \in\left(0, \mathcal{D}_{3}\right], t, \sigma \in(0, \infty)$ with $t>\sigma, \alpha \in \mathbb{N}_{0}^{3}$ with $|\alpha|_{1} \leq 1$. 
Proof. Let $\kappa \in\left(0, \mathcal{D}_{3}\right], y \in \partial \Omega, x \in \widetilde{\Omega}_{\kappa}$. If $x \notin \Omega$, there is $x^{\prime} \in \partial \Omega$ with $\left|x-x^{\prime}\right|=$ $\operatorname{dist}(x, \Omega)$, and we find with (9),

$$
\begin{aligned}
\left|x-y+\kappa \cdot m^{(\Omega)}(y)\right| & \geq\left|x^{\prime}-y+\kappa \cdot m^{(\Omega)}(y)\right|-\left|x-x^{\prime}\right| \\
& \geq \mathcal{D}_{2} \cdot \kappa-\operatorname{dist}(x, \Omega) \geq \mathcal{D}_{2} \cdot \kappa / 2,
\end{aligned}
$$

where the last inequality follows from the assumption $x \in \widetilde{\Omega}_{\kappa}$. If $x \in \Omega$, we recall that $y-\kappa \cdot m^{(\Omega)}(y) \notin \bar{\Omega}$ (see (10)), so there is $x^{\prime} \in \partial \Omega$ with

$$
\left|x-y+\kappa \cdot m^{(\Omega)}(y)\right| \geq\left|x^{\prime}-y+\kappa \cdot m^{(\Omega)}(y)\right|,
$$

hence again by (9): $\left|x-y+\kappa \cdot m^{(\Omega)}(y)\right| \geq \mathcal{D}_{2} \cdot \kappa$. Thus we get in any case,

$$
\left|x-y+\kappa \cdot m^{(\Omega)}(y)\right| \geq \mathcal{D}_{2} \cdot \kappa / 2 \text { for } \kappa \in\left(0, \mathcal{D}_{3}\right], y \in \partial \Omega, x \in \widetilde{\Omega}_{\kappa} .
$$

Take $\Phi$ as in (28). Then it follows from (31) and Lemma 2.4 that $\mathcal{V}^{(\tau, \kappa)}(\Phi)$ belongs to $C^{0}\left(\widetilde{\Omega}_{\kappa} \times(0, \infty)\right)^{3}$ with $\mathcal{V}^{(\tau, \kappa)}(\Phi)(\cdot, t) \in C^{1}\left(\widetilde{\Omega}_{\kappa}\right)^{3}$ for $t \in(0, \infty)$, and equation (29) holds. In particular,

$$
\mathcal{V}^{(\tau, \kappa)}(\Phi)\left|\overline{\Omega_{R_{0}}} \times(0, \infty) \in C^{0}\left(\overline{\Omega_{R_{0}}} \times(0, \infty)\right)^{3}, \quad \mathcal{V}^{(\tau, \kappa)}(\Phi)(\cdot, t)\right| \overline{\Omega_{R_{0}}} \in C^{1}\left(\overline{\Omega_{R_{0}}}\right)^{3}
$$

for $t \in(0, \infty)$. Now consider $\kappa \in\left(0, \mathcal{D}_{3}\right], y \in \partial \Omega, x \in \Omega_{R_{0}}$. If $|x-y| \leq \mathcal{D}_{2} \cdot \kappa / 2$, it follows with (31) that $\left|x-y+\kappa \cdot m^{(\Omega)}(y)\right| \geq|x-y|$. Next suppose that $2 \cdot \kappa \geq|x-y| \geq \mathcal{D}_{2} \cdot \kappa / 2$. Then we find by using (31) again,

$$
\left|x-y+\kappa \cdot m^{(\Omega)}(y)\right| \geq \mathcal{D}_{2} \cdot \kappa / 2 \geq\left(\mathcal{D}_{2} / 4\right) \cdot|x-y| .
$$

Finally, if $|x-y| \geq 2 \cdot \kappa$, it is obvious that $\left|x-y+\kappa \cdot m^{(\Omega)}(y)\right| \geq|x-y| / 2$. Thus we have in any case

$$
\left|x-y+\kappa \cdot m^{(\Omega)}(y)\right| \geq \min \left\{1 / 2, \mathcal{D}_{2} / 4\right\} \cdot|x-y| .
$$

Since $\left|x-y+\kappa \cdot m^{(\Omega)}(y)\right| \leq 2 \cdot R_{0}+\mathcal{D}_{3}$, we see that inequality (30) follows from (32) and Lemma 2.4 with $K=2 \cdot R_{0}+\mathcal{D}_{3}$.

Now we may carry out the

Proof of Theorem 2.4. Let $\Phi \in L^{2}\left(0, \infty, L^{p}(\partial \Omega)^{3}\right)$, for some $p \in(4 / 3,2]$. By (30), (29), Lemma 3.1 and Lebesgue's theorem on dominated convergence, we get that $\mathcal{V}_{\infty}^{(\tau)}(\Phi)(\cdot, t) \mid \Omega_{R_{0}} \in H^{1}\left(\Omega_{R_{0}}\right)^{3}$,

$$
\int_{\Omega_{R_{0}}}\left|\mathcal{V}^{(\tau, \kappa)}(\Phi)(x, t)-\mathcal{V}_{\infty}^{(\tau)}(\Phi)(x, t)\right|^{2} d x \rightarrow 0 \quad(\kappa \downarrow 0)
$$

for a.e. $t \in(0, \infty)$, and

$$
\int_{0}^{\infty} \int_{\Omega_{R_{0}}}\left|\nabla_{x} \mathcal{V}^{(\tau, \kappa)}(\Phi)(x, t)-\nabla_{x} \mathcal{V}_{\infty}^{(\tau)}(\Phi)(x, t)\right|^{2} d x \rightarrow 0 \quad \text { for } \kappa \downarrow 0 .
$$

As a consequence of $(34)$, we may choose a sequence $\left(\kappa_{n}\right)$ in $\left(0, \mathcal{D}_{3}\right]$ such that $\kappa_{n} \downarrow 0$ and

$$
\int_{\Omega_{R_{0}}}\left|\nabla_{x} \mathcal{V}^{\left(\tau, \kappa_{n}\right)}(\Phi)(x, t)-\nabla_{x} \mathcal{V}_{\infty}^{(\tau)}(\Phi)(x, t)\right|^{2} d x \rightarrow 0(n \rightarrow \infty) \text { for a.e. } t \in(0, \infty) \text {. }
$$


Fix some function $\zeta \in C_{0}^{\infty}\left(\mathbb{R}^{3}\right)$ with $\zeta \mid B_{R_{0} / 2}=1$, supp $(\zeta) \subset B_{R_{0}}$, and put

$$
\begin{aligned}
\mathcal{W}^{(\kappa)}(x, t) & :=\zeta(x) \cdot \mathcal{V}^{(\tau, \kappa)}(\Phi)(x, t) \quad \text { for } x \in \overline{\Omega_{R_{0}}}, t \in(0, \infty), \\
\mathcal{W}(x, t) & :=\zeta(x) \cdot \mathcal{V}_{\infty}^{(\tau)}(\Phi)(x, t) \quad \text { for } x \in \Omega_{R_{0}}, t \in(0, \infty) .
\end{aligned}
$$

Then $\mathcal{W}^{(\kappa)}(\cdot, t) \in C^{1}\left(\overline{\Omega_{R_{0}}}\right)^{3}$ for $\kappa \in\left(0, \mathcal{D}_{3}\right], t \in(0, \infty)$ (see Lemma 3.4$), \mathcal{W}(\cdot, t) \in$ $H^{1}\left(\Omega_{R_{0}}\right)$,

$$
\left\|\mathcal{W}^{\left(\kappa_{n}\right)}(\cdot, t)-\mathcal{W}(\cdot, t)\right\|_{1,2} \rightarrow 0 \quad(n \rightarrow \infty) \text { for a.e. } t \in(0, \infty)
$$

(see (33), (35)). Therefore

$$
\left\|\mathcal{W}^{\left(\kappa_{n}\right)}(\cdot, t)|\partial \Omega-\operatorname{trace}(\mathcal{W}(\cdot, t))| \partial \Omega\right\|_{2} \rightarrow 0 \quad(n \rightarrow \infty) \quad \text { for a.e. } t \in(0, \infty) .
$$

Since $\zeta \mid \partial \Omega=1$, this means

$$
\left\|\mathcal{V}^{\left(\tau, \kappa_{n}\right)}(\Phi)(\cdot, t)|\partial \Omega-\operatorname{trace}(\mathcal{W}(\cdot, t))| \partial \Omega\right\|_{2} \rightarrow 0 \quad(n \rightarrow \infty)
$$

for a.e. $t \in(0, \infty)$. On the other hand, take $T \in(0, \infty)$. Then

$$
\begin{aligned}
& \int_{0}^{T} \int_{\partial \Omega} \int_{0}^{t} \int_{\partial \Omega}\left(|x-y|^{2}+t-\sigma\right)^{-3 / 2} \cdot|\Phi(y, \sigma)| d \Omega(y) d \sigma d \Omega(x) d t \\
& =\int_{0}^{T} \int_{\partial \Omega} \int_{\sigma}^{T} \int_{\partial \Omega}\left(|x-y|^{2}+t-\sigma\right)^{-3 / 2} d \Omega(x) d t \cdot|\Phi(y, \sigma)| d \Omega(y) d \sigma \\
& \leq \mathcal{C} \cdot T^{1 / 2} \cdot\left\|\Phi \mid S_{T}\right\|_{1} \leq \mathcal{C} \cdot T \cdot\|\phi\|_{L^{2}\left(0, \infty, L^{p}(\partial \Omega)^{3}\right)} .
\end{aligned}
$$

It follows from (30) and Lebesgue's theorem on dominated convergence that

$$
\int_{0}^{T} \int_{\partial \Omega}\left|\mathcal{V}^{(\tau, \kappa)}(\Phi)(x, t)-\mathcal{V}_{\infty}^{(\tau)}(\Phi)(x, t)\right| d \Omega(x) d t \rightarrow 0 \quad(\kappa \downarrow 0) .
$$

Thus we may conclude there is a subsequence $\left(\widetilde{\kappa}_{n}\right)$ of $\left(\kappa_{n}\right)$ such that

$$
\mathcal{V}^{\left(\tau, \widetilde{\kappa}_{n}\right)}(\Phi)(x, t)-\mathcal{V}_{\infty}^{(\tau)}(\Phi)(x, t) \rightarrow 0 \quad(n \rightarrow \infty) \text { for a.e. } t \in(0, T) \text { and a.e. } x \in \partial \Omega \text {. }
$$

In view of $(36)$, it follows that $\operatorname{trace}(\mathcal{W}(\cdot, t))\left|\partial \Omega=\mathcal{V}_{\infty}^{(\tau)}(\Phi)(\cdot, t)\right| \partial \Omega$ for a.e. $t \in(0, T)$. Since $T$ was chosen arbitrarily in $(0, \infty)$, the preceding equation even holds for a.e. $t \in$ $(0, \infty)$. But $\mathcal{W}(\cdot, t)=\zeta \cdot \mathcal{V}_{\infty}^{(\tau)}(\Phi)(\cdot, t) \mid \Omega_{R_{0}}$ for $t \in(0, \infty)$, and $\zeta\left|B_{R_{0} / 2}=1, \zeta\right| B_{R_{0}^{c}}=0$ so Theorem 2.4 is proved.

\section{References}

[1] R. A. Adams and J. J. F. Fournier, Sobolev Spaces, 2nd ed., Academic Press, Amsterdam, 2003.

[2] P. Deuring, The single-layer potential associated with the time-dependent Oseen system, in: Proceedings of the 2006 IASME/WSEAS International Conference on Continuum Mechanics (Chalkida, 2006), 117-125.

[3] P. Deuring and S. Kracmar, Exterior stationary Navier-Stokes flows in 3D with non-zero velocity at infinity: approximation by flows in bounded domains, Math. Nachr. 269-270 (2004), 86-115.

[4] Y. Enomoto and Y. Shibata, Local energy decay of solutions to the Oseen equation in exterior domain, Indiana Univ. Math. J. 53 (2004), 1291-1330. 
[5] Y. Enomoto and Y. Shibata, On the rate of decay of the Oseen semigroup in exterior domains and its application to Navier-Stokes equation, J. Math. Fluid Mech. 7 (2005), 339-367.

[6] R. Farwig, A variational approach in weighted Sobolev spaces to the operator $-\Delta+\partial / \partial x_{1}$ in exterior domains in $\mathbb{R}^{3}$, Math. Z. 210 (1992), 449-464.

[7] S. Fučik, O. John and A. Kufner, Function Spaces, Noordhoff, Leyden, 1977.

[8] Y. Kobayashi and Y. Shibata, On the Oseen equation in three dimensional exterior domains, Math. Ann. 310 (1998), 1-45.

[9] J. Nečas, Les méthodes directes en théorie des équations elliptiques, Masson, Paris, 1967.

[10] Y. Shibata, On an exterior initial boundary value problem for Navier-Stokes equations, Quarterly Appl. Math. 57 (1999), 117-155.

[11] Z. Shen, Boundary value problems for parabolic Lamé systems and a nonstationary linearized system of Navier-Stokes equations in Lipschitz cylinders, American J. Math. 113 (1991), 293-373.

[12] E. Stein, Singular Integrals and Differentiability Properties of Functions, Princeton University Press, Princeton, N.J., 1970.

[13] R. Temam, Navier-Stokes Equations. Theory and Numerical Analysis, AMS Chelsea Publishing, Providence, R.I., 2001. 